\title{
Complexity of Jackiw-Teitelboim gravity
}

\author{
Adam R. Brown, ${ }^{1,2}$ Hrant Gharibyan, ${ }^{1}$ Henry W. Lin, ${ }^{3}$ Leonard Susskind, ${ }^{1}$ \\ Lárus Thorlacius, ${ }^{1,4}$ and Ying Zhao ${ }^{1,5}$ \\ ${ }^{1}$ Stanford Institute for Theoretical Physics and Department of Physics, Stanford University, \\ Stanford, California 94305, USA \\ ${ }^{2}$ Google, Mountain View, California 94043, USA \\ ${ }^{3}$ Department of Physics, Princeton University, Princeton, New Jersey 08540, USA \\ ${ }^{4}$ University of Iceland, Science Institute, Dunhaga 3, 107 Reykjavik, Iceland \\ and The Oskar Klein Centre for Cosmoparticle Physics, Department of Physics, Stockholm University, \\ AlbaNova, 10691 Stockholm, Sweden \\ ${ }^{5}$ Institute for Advanced Study, Princeton, New Jersey 08540, USA
}

(Received 8 January 2019; published 25 February 2019)

\begin{abstract}
The Jackiw-Teitelboim (JT) model arises from the dimensional reduction of charged black holes. Motivated by the holographic complexity conjecture, we calculate the late-time rate of change of action of a Wheeler-DeWitt patch in the JT theory. Surprisingly, the rate vanishes. This is puzzling because it contradicts both holographic expectations for the rate of complexification and also action calculations for charged black holes. We trace the discrepancy to an improper treatment of boundary terms when naively doing the dimensional reduction. Once the boundary term is corrected, we find exact agreement with expectations. We comment on the general lessons that this might hold for holographic complexity and beyond.
\end{abstract}

DOI: 10.1103/PhysRevD.99.046016

\section{INTRODUCTION}

The Jackiw-Teitelboim (JT) model of 1 + 1-dimensional dilaton gravity $[1,2]$ is useful for studying conjectures relating the geometry of black holes and scrambling in dual quantum systems. This simple model holographically reproduces the nearly conformal dynamics [3] of the Sachdev-Ye-Kitaev (SYK) model [4,5] at low energies. In the present paper, we will focus on a particular aspect of the duality-the conjectured relationship between quantum complexity and emergent spacetime.

The SYK model is a good place to test the conjectured relation between complexity and the size of wormholes [6-8]. For one thing the SYK model is, from the beginning, a theory of (fermionic) qubits, which means that in principle the definition of complexity could be fairly standard. On the other hand, the gravitational dual at sufficiently low energies is fairly well understood.

In particular, the low-energy dynamics of the SYK model is characterized by a spontaneously and explicitly

Published by the American Physical Society under the terms of the Creative Commons Attribution 4.0 International license. Further distribution of this work must maintain attribution to the author(s) and the published article's title, journal citation, and DOI. Funded by SCOAP . broken reparametrization symmetry, with the effective action of the associated Nambu-Goldstone modes given by the Schwarzian [9-11]. The resulting universal dynamics exhibits a number of distinctive features, including outof-time-order four-point functions that saturate the chaos bound [12]. The boundary dynamics of 1+1-dimensional JT dilaton gravity is governed by the same broken timereparametrization symmetry and Schwarzian action, motivating its identification as a bulk dual for low-energy SYK dynamics. At the same time, JT theory is simple enough to allow explicit computations of both the volume of a maximal surface and the action of a Wheeler-DeWitt (WDW) patch. This gives us the opportunity to test both the complexity-volume $(\mathrm{CV})$ duality and complexity-action (CA) duality against expectations from the quantum-chaotic nature of the SYK model.

For most purposes, JT theory is the dimensional reduction of the near-horizon dynamics of a nearextremal Reissner-Nordström (RN) black hole of Einstein-Maxwell theory, and one might think they give identical results for holographic complexity. Indeed for CV duality JT and RN agree with each other, and with the expected behavior of complexity for SYK quantum mechanics. Surprisingly, when we compute the action in WDW patches we do not find agreement. The late-time rate of growth of action for the JT theory vanishes, in 
disagreement with our expectations for the growth of complexity for chaotic systems like SYK. ${ }^{1}$

At first sight, this seems to be a serious counterexample to the CA conjecture. On the other hand when the EinsteinMaxwell action is computed for the near-extremal RN black hole, we find perfect agreement with the expectations for complexity growth. By carefully constructing the dimensional reduction of $\mathrm{RN}$ we have been able to trace the origin of the discrepancy. In eliminating the gauge field from $\mathrm{RN}$, the dimensional reduction inadvertently introduces an inappropriate boundary term at the edge of the WDW patch. There does not seem to be a consistent way to remove this term without bringing back the gauge field as a degree of freedom (d.o.f.).

We emphasize that for the purposes for which it has been used up till now the JT model correctly reflects much of the physics of the SYK model, including the lowenergy dynamics governed by broken reparametrization symmetry at low temperature. We find, however, that for more subtle quantities, such as holographic complexity, the standard formulation of the JT model as a theory of two-dimensional dilaton gravity fails to give sensible results, whereas the original higher-dimensional EinsteinMaxwell theory succeeds.

The paper is organized as follows. In Sec. II we set the stage for the rest of the discussion by reviewing salient features of the near-extremal limit of electrically charged RN black holes in 3+1-dimensional Einstein-Maxwell theory. In Sec. III we write down the action of the JT model and describe its black hole solutions. This serves to fix notation and set up a simple $1+1$-dimensional framework where explicit calculations can be carried out to test proposals for holographic complexity. CV duality for JT black holes is considered in Sec. IV and found to reproduce predictions for higher-dimensional near-extremal RN black holes. In Sec. V we turn our attention to CA duality and find that it apparently runs into a problem in the JT theory. In Sec. VI we take steps to resolve the problem by reexamining how JT gravity arises as an effective theory for low-energy radial modes in the near-horizon region of a near-extremal RN black hole. The eventual resolution involves a careful treatment of boundary terms in the action and is presented in Sec. VII. Further examples of systems where boundary terms play similar roles are presented in the Appendixes.

\section{COMPLEXITY=ACTION FOR RN BLACK HOLES}

We begin our discussion by recalling some facts about charged black holes in $3+1$-dimensional Einstein-Maxwell theory with action

\footnotetext{
${ }^{1}$ Throughout this paper, "late time" refers to much longer than the thermal timescale but less than the exponential timescale on which complexity is expected to saturate and on which classical geometry no longer provides a reliable description [6].
}

$$
\begin{aligned}
\mathcal{S}= & \frac{1}{16 \pi} \int_{\mathcal{M}} d^{4} x \sqrt{-g}\left(\frac{1}{\ell^{2}} R-F_{\mu \nu} F^{\mu \nu}\right) \\
& +\frac{1}{8 \pi \ell^{2}} \int_{\partial \mathcal{M}} d^{3} y \sqrt{-h}\left(K-K_{0}\right),
\end{aligned}
$$

where $\ell \equiv \sqrt{G_{N}}$ is the $3+1$-dimensional Planck length. This action describes an electromagnetic field gravitationally backreacting on curved spacetime. It includes the usual Gibbons-Hawking-York boundary term [13,14] involving the trace of the extrinsic curvature $K$ at an asymptotic spacetime boundary with induced metric $h_{i j}$. It also includes a regulator term that subtracts $K_{0}$, the trace of the extrinsic curvature of the same boundary surface when embedded in a flat spacetime, in order to obtain a finite free energy from the corresponding on-shell Euclidean action. ${ }^{2}$

The boundary conditions obeyed by the electromagnetic field at $\partial \mathcal{M}$ will play an important role in our discussion. As it stands, the action (2.1) does not include any boundary term involving the Maxwell field and $A_{\mu}$ is kept fixed at the boundary. In the Euclidean formalism this corresponds to a thermal ensemble where the chemical potential is held fixed but the total electric charge of the system is allowed to fluctuate. If, on the other hand, the following boundary term is added to the action,

$$
\mathcal{S}_{b}^{\mathrm{em}}=\frac{1}{4 \pi} \int_{\partial \mathcal{M}} d^{3} y \sqrt{-h} \hat{n}_{\mu} F^{\mu \nu} A_{\nu}
$$

then free variations of $A_{\mu}$ are allowed at the boundary and the corresponding thermal ensemble is that of fixed charge but varying chemical potential. We will revisit $3+1$ dimensional Maxwell boundary terms in Appendix C.

A notable solution for the action (2.1) is the spherically symmetric Reissner-Nordström black hole with electric charge $Q>0$ and mass $M \geq Q / \ell$, described by

$$
\begin{aligned}
d s^{2} & =-f(r) d t^{2}+\frac{d r^{2}}{f(r)}+r^{2} d \Omega^{2}, \\
f(r) & =\left(1-\frac{r_{+}}{r}\right)\left(1-\frac{r_{-}}{r}\right), \\
F_{r t} & =\frac{Q}{r^{2}},
\end{aligned}
$$

where $r_{ \pm}=\ell^{2} M \pm \sqrt{\ell^{4} M^{2}-\ell^{2} Q^{2}}$ are the locations of the outer and inner horizon.

We will be interested in these black holes when they are very near to extremality, $M \rightarrow M_{0}=Q / \ell$. In the extremal

\footnotetext{
${ }^{2}$ Alternatively, one can introduce a small negative cosmological constant into the $3+1$-dimensional theory and include the standard boundary counterterms that render the free energy finite.
} 


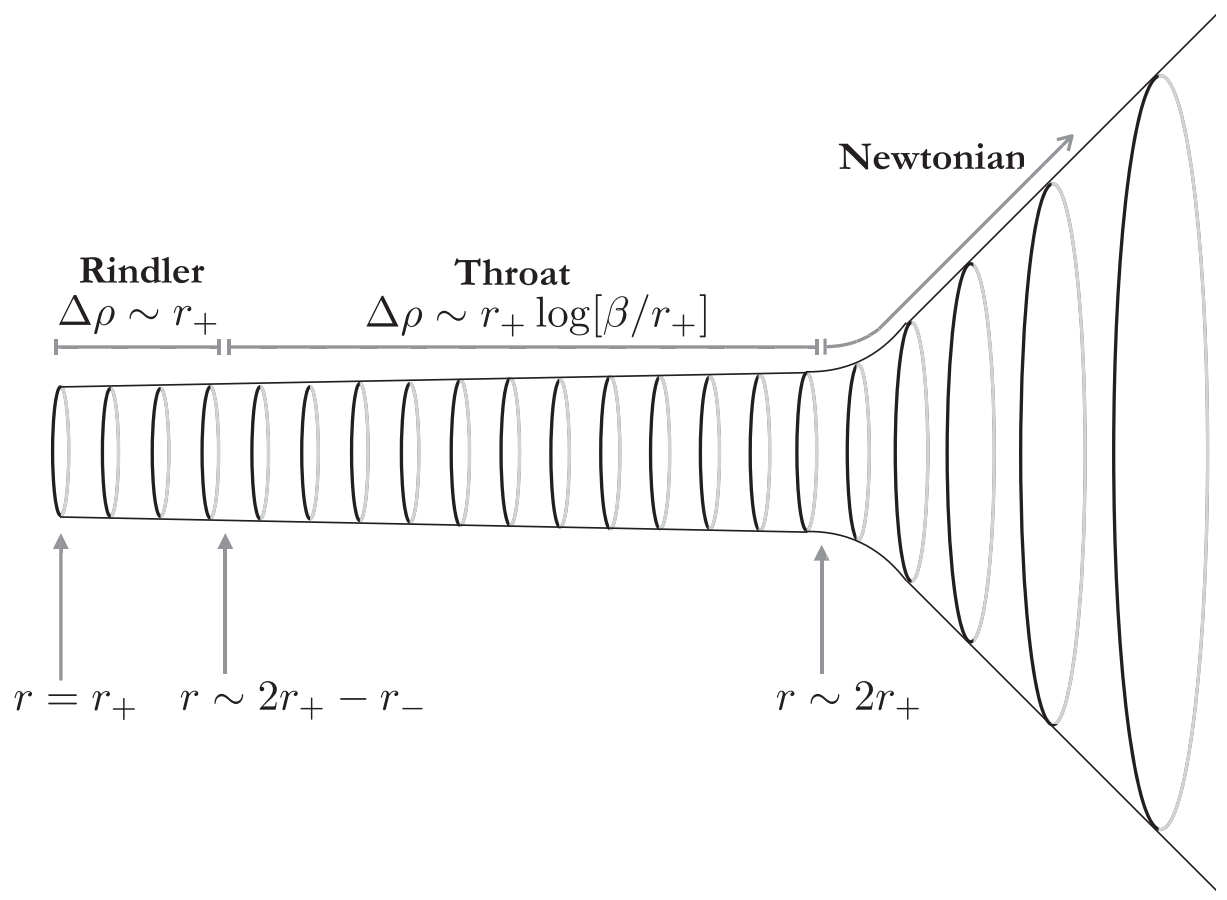

FIG. 1. The three regions outside the horizon of a near-extremal RN black hole. The transition from throat to Newtonian region occurs at $r \sim 2 r_{+}$, which is also the approximate location of the top of the potential barrier [15], and also the approximate location of the curved JT boundary in Fig. 5.

limit, the horizons are degenerate $r_{+}=r_{-}=\ell Q$ and the Hawking temperature,

$$
T=\frac{\left(r_{+}-r_{-}\right)}{4 \pi r_{+}^{2}},
$$

goes to zero. Thus for near-extremal black holes $r_{+}-$ $r_{-} \ll r_{+}$, or equivalently $\beta \gg r_{+}$. Following our recent work [15], we find it useful to divide the spacetime geometry outside a near-extremal black hole into the three regions shown in Fig. 1:

(i) Closest to the outer horizon of the black hole is the Rindler region, $r_{+}<r \lesssim 2 r_{+}-r_{-}$, where the proper distance from the horizon, $\Delta \rho=\int_{r_{+}}^{r} d r^{\prime} /$ $\sqrt{f\left(r^{\prime}\right)}$, is in the range

$$
0<\Delta \rho \lesssim r_{+}
$$

(ii) The "throat" region, $2 r_{+}-r_{-} \lesssim r \lesssim 2 r_{+}$, becomes long in the low-temperature limit,

$$
r_{+} \lesssim \Delta \rho \lesssim r_{+} \log \left(\frac{\beta}{r_{+}}\right)
$$

and in this case the spacetime geometry is well approximated locally by $\operatorname{AdS}_{2} \times S^{2}$. The long throat supports a low-energy sector of long-wavelength radial excitations that are described by an effective two-dimensional theory, the Jackiw-Teitelboim model, discussed in Sec. III below.

(iii) Finally, there is the Newtonian region, $2 r_{+} \lesssim r$, where the metric approaches that of flat spacetime $f(r) \approx 1$. The JT boundary from Fig. 5 may be identified with where the $\mathrm{AdS}_{2}$ throat meets the Newtonian region.

In $[7,8]$, the late-time rate of change of the action of a WDW patch for RN black holes was calculated to be

$$
\frac{d S}{d\left(t_{L}+r_{R}\right)}=\frac{Q^{2}}{r_{-}}-\frac{Q^{2}}{r_{+}}=4 S T
$$

where $T$ is the Hawking temperature (2.4) and $S=\pi r_{+}^{2} / \ell^{2}$ is the Bekenstein-Hawking entropy. For our purposes, the essential aspect of this result is that the action advances linearly at late times. This is consistent with expectations for complexity - at preexponential times, a generic quantum system will complexify with a constant rate. However, it is superficially inconsistent with the vanishing rate of action advance that we obtain for the JT theory in Sec. V. This inconsistency will be resolved in Sec. VII, in favor of Eq. (2.7).

\section{JACKIW-TEITELBOIM MODEL}

We now turn to our main object of study, the JT model of $1+1$-dimensional dilaton gravity $[1,2]$. We start by introducing the basic equations of the model and write down a 
family of static solutions. This serves to establish notation ${ }^{3}$ and sets the stage for our subsequent calculations testing the $\mathrm{CV}$ and $\mathrm{CA}$ conjectures.

The fields of the JT model consist of a metric and a realvalued dilaton field, defined on a 1+1-dimensional manifold $\mathcal{M}$ with timelike boundary $\partial \mathcal{M}$. By adopting a suitable field redefinition, the action can be expressed in a simple form, with no derivatives acting on the dilaton,

$$
\begin{aligned}
\mathcal{S}_{J T}= & \frac{\varphi_{0}}{2}\left(\int_{\mathcal{M}} d^{2} x \sqrt{-g} R+2 \int_{\partial \mathcal{M}} d \tau K\right) \\
& +\frac{1}{2} \int_{\mathcal{M}} d^{2} x \sqrt{-g} \varphi\left(R+\frac{2}{L^{2}}\right) \\
& +\int_{\partial \mathcal{M}} d \tau \varphi\left(K-\frac{1}{L}\right),
\end{aligned}
$$

where $L$ is a characteristic length scale of the model.

The boundary terms in the action involving the extrinsic curvature are needed to make the gravitational variational problem well defined and we have also included a boundary counterterm that renders the Euclidean on-shell action finite. The terms on the first line are independent of the dilaton field $\varphi$ and are instead multiplied by a constant $\varphi_{0}$. Their sum is proportional to a topological invariant, the Euler character of the 1+1-dimensional manifold, and thus they do not contribute to the equations of motion. They do, however, contribute to thermodynamic quantities such as the free energy and entropy of the black hole. As we shall see in Sec. VI below, this model, including the topological terms, arises naturally when one considers the spherical reduction of 3+1-dimensional Einstein-Maxwell theory around near-extremal charged black holes. In this context, the higher-dimensional black hole charge $Q$ determines the constant $\varphi_{0}$ in front of the topological terms via the relation $\varphi_{0}=Q^{2} / 2$. For now, we want to explore the JT theory on its own terms as a $1+1$-dimensional model and for this purpose we can simply assume that $\varphi_{0} \gg 1$ but leave it otherwise undetermined.

The boundary of the $1+1$-dimensional spacetime is taken to be along a curve of constant dilaton field, $\left.\varphi\right|_{\partial M}=\varphi_{B}$, and the integration variable $\tau$ in the boundary terms in the action is the proper time along this boundary curve. We can introduce a separation of scales in the JT model by imposing the condition $\varphi_{B} \ll \varphi_{0}$. For the time being, we impose this by hand, but in the higherdimensional context it amounts to restricting the range of the 1+1-dimensional effective description to lie well inside the throat region of a near-extremal ReissnerNordström black hole.

\footnotetext{
${ }^{3}$ For the most part, our conventions in this section follow those of [3].
}

\section{A. AdS $_{2}$ geometry and JT black holes}

The action (3.1) gives rise to the following field equations,

$$
\begin{gathered}
0=R+\frac{2}{L^{2}} \\
0=\nabla_{\alpha} \nabla_{\beta} \varphi-g_{\alpha \beta}\left(\nabla^{2} \varphi-\frac{1}{L^{2}} \varphi\right) .
\end{gathered}
$$

The variational equation for the dilaton field (3.2) implies that the spacetime geometry is always locally $\mathrm{AdS}_{2}$. We find it convenient to use global coordinates $(\nu, \sigma)$, where the $\mathrm{AdS}_{2}$ metric takes the form

$$
d s^{2}=\frac{L^{2}}{\sin ^{2} \sigma}\left(-d \nu^{2}+d \sigma^{2}\right) .
$$

The full range of the coordinates is $-\infty<\nu<\infty$ and $0<\sigma<\pi$, but when we take the dilaton field into consideration the coordinate range is restricted by the boundary at $\varphi=\varphi_{B}$. This is best illustrated by considering a specific field configuration.

A one-parameter family of solutions to the Einstein equations (3.3) is given by

$$
\varphi(\nu, \sigma)=\varphi_{H} \frac{\cos \nu}{\sin \sigma},
$$

where the integration constant $\varphi_{H}>0$ is the value of $\varphi$ on the event horizon of the two-dimensional black hole, as we will see shortly. The boundary at $\varphi=\varphi_{B}$ is located on a set of timelike curves,

$$
\sin \sigma=\varepsilon \cos \nu,
$$

with $\varepsilon=\varphi_{H} / \varphi_{B}$, that intersect the $\mathrm{AdS}_{2}$ boundary at $\nu= \pm \frac{\pi}{2}$, as shown in Fig. 2. In the following we assume that $\varepsilon \ll 1$ and work to leading order in powers of $\varepsilon$.

The dilaton field $\varphi$ in (3.5) is periodic in the global time $\nu$. It is negative in the range $\frac{\pi}{2}<\nu<\frac{3 \pi}{2}$ and becomes arbitrarily negative as $\sigma \rightarrow 0$ or $\sigma \rightarrow \pi$. This means that $\Phi \equiv \varphi_{0}+\varphi$ goes to zero along another set of timelike curves (also shown in Fig. 2). In the spherical reduction from $3+1$ dimensions discussed in Sec. VI, the field $\Phi$ is proportional to the area of the transverse two-sphere and with the higher-dimensional interpretation in mind it is natural to view the $\Phi=0$ curves as singularities. The associated event horizon is along the diagonal lines $\nu=$ $\pm\left(\sigma-\frac{\pi}{2}\right)$ and it is easily checked that $\varphi=\varphi_{H}$ everywhere on the horizon. We note that the spherically reduced Reissner-Nordström solution, given in (6.10) below, indeed has a timelike curvature singularity precisely where $\Phi$ vanishes. However, the small $\varphi$ truncation that leads to the field equations of the JT model breaks down long before $\varphi$ 


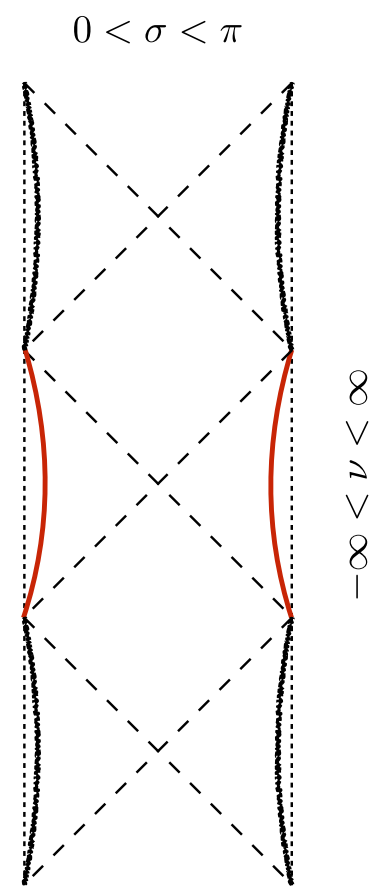

FIG. 2. Jackiw-Teitelboim black hole in global $\mathrm{AdS}_{2}$ coordinates. The red curves indicate the outer boundary of the $1+1$ dimensional black hole spacetime, while the solid black curves indicate singularities where $\Phi=0$. The dashed diagonal lines show the location of the black hole horizon.

approaches $-Q^{2} / 2$ so the $\Phi=0$ curve of the JT model is only a proxy for the true physical singularity.

\section{B. JT black hole thermodynamics}

The relationship between $\Phi$ and the area of the transverse two-sphere will be derived in Sec. VI and is given in (6.1). The corresponding entropy of the $1+1$-dimensional black hole is

$$
S=\left.2 \pi \Phi\right|_{\text {Horizon }}=2 \pi \varphi_{0}+2 \pi \varphi_{H} .
$$

This entropy assignment can of course be made without giving it any higher-dimensional interpretation but in that case it would appear rather arbitrary. The entropy of an extremal 3+1-dimensional Reissner-Nordström black hole is $S_{0}=\pi Q^{2}=2 \pi \varphi_{0}$ so we see that $2 \pi \varphi_{H}$ amounts to the added entropy of a near-extremal black hole with the same charge.

The black hole character of the solution (3.5) is more immediately apparent when we write it in a $1+1$-dimensional version of Schwarzschild coordinates,

$$
d s^{2}=-\frac{r^{2}-r_{H}^{2}}{L^{2}} d t^{2}+\frac{L^{2}}{r^{2}-r_{H}^{2}} d r^{2} .
$$

In this coordinate system, there is a coordinate singularity at the event horizon at $r=r_{H}$ and the dilaton is linear in $r$,

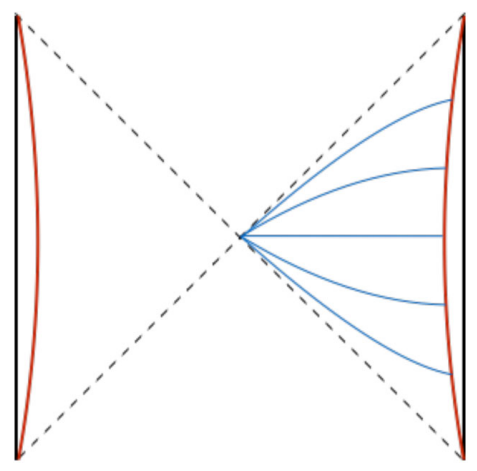

FIG. 3. Jackiw-Teitelboim black hole. Curves of constant Schwarzschild time outside the event horizon are shown in blue.

$$
\varphi=\varphi_{H} \frac{r}{r_{H}} .
$$

The requirement that the Euclidean continuation of the metric (3.8) be smooth at $r=r_{H}$ yields the following Hawking temperature for the 1+1-dimensional black hole:

$$
T=\frac{r_{H}}{2 \pi L^{2}} .
$$

It is straightforward to work out the Kruskal extension of the Schwarzschild form of the $\mathrm{AdS}_{2}$ metric (3.8) and find an explicit coordinate transformation relating $(\nu, \sigma)$ and $(t, r)$. In particular, the relationship between the global time and Schwarzschild time along the boundary curve at $r=r_{B}$ is

$$
\tan \left(\frac{\nu}{2}+\frac{\pi}{4}\right)=e^{2 \pi T t}
$$

up to $O\left(\varepsilon^{2}\right)$ correction terms that are small in the limit $r_{B} \gg r_{H}$. Slices of constant Schwarzschild time are shown in Fig. 3.

The on-shell Euclidean action can be obtained from the Euclidean version of (3.1) including all the boundary terms. A straightforward calculation gives

$$
\mathcal{S}_{E}=-S+\beta E
$$

where

$$
\begin{gathered}
S=2 \pi \varphi_{0}+4 \pi^{2} L^{2} \frac{\varphi_{B}}{r_{B}} T, \\
E=2 \pi^{2} L^{2} \frac{\varphi_{B}}{r_{B}} T^{2},
\end{gathered}
$$

which has the form of a free energy of a near-extremal Reissner-Nordström black hole in a fixed charge ensemble (see Appendix B). By comparing with (B3) we identify $S$ as the $3+1$-dimensional black hole entropy and $E$ as the 
added mass of the near-extremal black hole compared to the extremal mass. Furthermore, by equating the coefficients of $T$ and $T^{2}$ in $S$ and $E$, respectively, of the 1+1-dimensional black hole to the corresponding terms in (B3) one finds that $\varphi(r)=r / \ell$, where $\ell$ is the $3+1$-dimensional Planck length.

\section{COMPLEXITY=VOLUME IN THE JT MODEL}

We are now in a position to test proposals for the holographic dual of quantum complexity in the simplified setting of 1+1-dimensional dilaton gravity. We start with the complexity-volume (CV) duality and then consider the complexity-action (CA) duality in Sec. V. The CV proposal [6] states that the complexity of the quantum state dual to the black hole is proportional to the spatial volume of a maximal slice behind the horizon,

$$
\mathcal{C} \sim \frac{V}{G \ell_{0}},
$$

where $G$ is Newton's constant and $\ell_{0}$ is a characteristic length scale associated with the black hole in question. In the case at hand, the "volume" of a maximal slice is simply the length $L_{s}$ of a spacelike geodesic and the characteristic length scale $\ell_{0}$ can be taken to be the $\operatorname{AdS}_{2}$ scale $L$. There is, however, no notion of Newton's constant that is intrinsic to $1+1$-dimensional gravity and we need to adapt the prescription accordingly. Our proposal is to include a factor of $\varphi_{0}$ in the proportionality factor between complexity and volume in the JT model,

$$
\mathcal{C} \sim \frac{\varphi_{0} L_{s}}{L} .
$$

This is motivated by the general expectation that the complexity should grow at a rate that is proportional to the number of d.o.f. of the dual quantum system. The number of d.o.f. is in turn proportional to the black hole entropy and for our $1+1$-dimensional black holes the

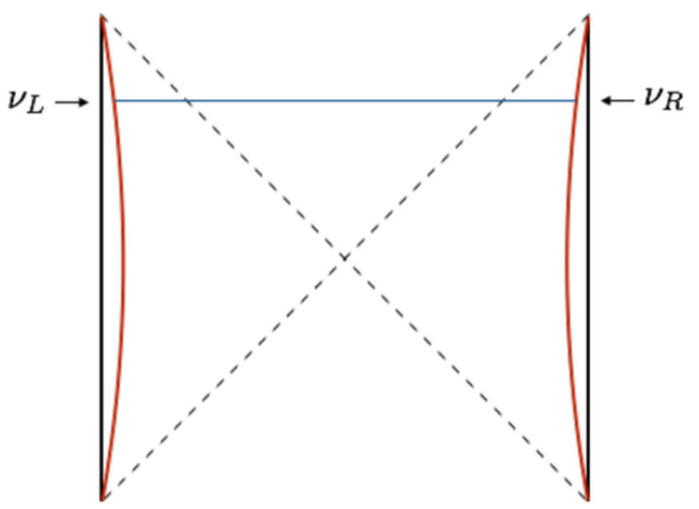

entropy is dominated by the extremal entropy, $S_{0}=2 \pi \varphi_{0}$. This is of course also intimately related to the higherdimensional origins of the Jackiw-Teitelboim model. Under spherical reduction, each 1+1-dimensional event is accompanied by a transverse sphere, whose area in Planck units is given by the dilaton field, and from this point of view the $1+1$-dimensional definition (4.2) is a special case of (4.1).

Let us first consider a maximal slice of the form shown in the left panel of Fig. 4, where the spacelike geodesic connects boundary points that have the same value of global time, $\nu_{L}=\nu_{R} \equiv \nu_{0}$. The complexity is proportional to the proper length that lies inside the horizon,

$$
\begin{aligned}
L_{s} & =L \int_{\frac{\pi}{2}-\nu_{0}}^{\frac{\pi}{2}+\nu_{0}} \frac{d \sigma}{\sin \sigma} \\
& =2 L \log \left(\tan \left(\frac{\nu_{0}}{2}+\frac{\pi}{4}\right)\right) \\
& \approx 4 \pi L T t_{0},
\end{aligned}
$$

where in the final step we have used (3.11) to convert from global time to Schwarzschild time. It then immediately follows from (4.2) that complexity-as-volume grows linearly with Schwarzschild time in the JT model,

$$
C \sim \phi_{0} T t
$$

and the rate of growth is proportional to the black hole temperature $d C / d t \sim S T$.

More generally, we can consider a spacelike geodesic that connects left- and right-hand boundary points at different global time, $\nu_{L} \neq \nu_{R}$, as shown in the right-hand panel of Fig. 4. A geodesic satisfying these boundary conditions [up to $O\left(\varepsilon^{2}\right)$ corrections] is given by the following curve in the $(\nu, \sigma)$ plane,

$$
\sin \left(\nu-\nu_{+}\right)=\sin \nu_{-} \cos \sigma,
$$

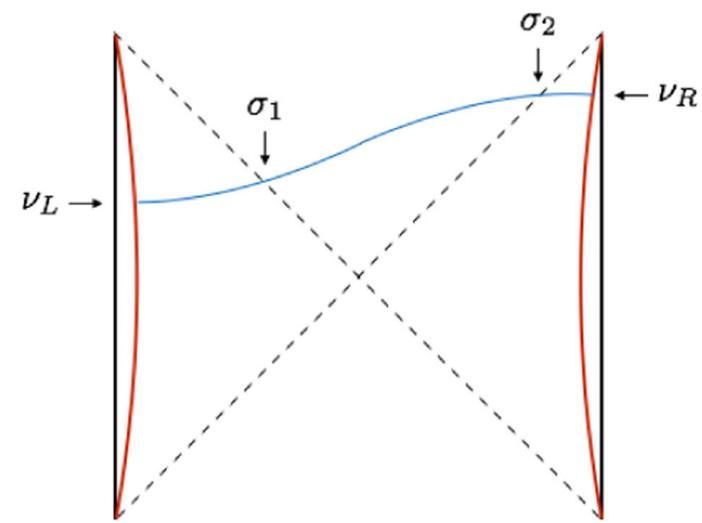

FIG. 4. Minimal slices for $C=V$ calculation. The left panel shows a "horizontal" slice with $\nu_{L}=\nu_{R}$. On the right, a more general slice with independent values of $\nu_{L}, \nu_{R}$. 
where $\nu_{ \pm}=\frac{1}{2}\left(\nu_{L} \pm \nu_{R}\right)$. The geodesic meets the horizon $\sigma=\frac{\pi}{2} \pm \nu$ at two intersection points at $\sigma=\sigma_{1}$ and $\sigma=\sigma_{2}$ as indicated in Fig. 4. The locations of the intersection points are easily obtained from the geodesic curve and are given by the relations

$$
\begin{aligned}
\tan \sigma_{1} & =\frac{\left(\cos \nu_{+}-\sin \nu_{-}\right)}{\sin \nu_{+}} ; \\
\tan \sigma_{2} & =-\frac{\left(\cos \nu_{+}+\sin \nu_{-}\right)}{\sin \nu_{+}}
\end{aligned}
$$

The geodesic length inside the horizon is then given by the integral

$$
L_{s}=L \int_{\sigma_{1}}^{\sigma_{2}} \frac{d \sigma}{\sin \sigma} \sqrt{1-\left(\frac{d \nu}{d \sigma}\right)^{2}}
$$

with $\nu(\sigma)$ obtained from (4.5). This yields a relatively simple closed form expression involving $\sigma_{1}$ and $\sigma_{2}$,

$$
\begin{aligned}
L_{s}= & \frac{L}{2}\left[\log \left(\frac{1-\cos \sigma}{1+\cos \sigma}\right)\right. \\
& \left.+\log \left(\frac{1+\sin ^{2} \nu_{-} \cos \sigma+\cos \nu_{-} \sqrt{1-\sin ^{2} \nu_{-} \cos ^{2} \sigma}}{1-\sin ^{2} \nu_{-} \cos \sigma+\cos \nu_{-} \sqrt{1-\sin ^{2} \nu_{-} \cos ^{2} \sigma}}\right)\right]_{\sigma_{1}}^{\sigma_{2}},
\end{aligned}
$$

which can in turn be expressed in terms of $\nu_{L}$ and $\nu_{R}$ via (4.6).

As a side note, in the Schwarzian regime [3] this formula simplifies when the usual Schwarzian variables $\nu_{L}(u)$ and $\nu_{R}(u)$ are used, where $u$ is the proper time along the boundary trajectories:

$$
L_{s}=-\log \left[\frac{\nu_{L}^{\prime}(u) \nu_{R}^{\prime}(u)}{\cos ^{2}\left(\frac{\nu_{L}-\nu_{R}}{2}\right)}\right]+\mathcal{O}\left(\epsilon^{2}\right)
$$

In this expression, we have dropped the constant IR regulator $\sim \log \epsilon^{2}$. We see explicitly that the volume is invariant under $S L(2, R)$ transformations. In fact, we could have guessed the form of this expression based on $S L(2, R)$ symmetry and the fact that the length is only a function of $\nu$ and its first derivative.

We are mainly interested in the late-time limit on one boundary, i.e., $\nu_{R} \rightarrow \frac{\pi}{2}$ while keeping $\nu_{L}$ fixed. From Fig. 4 it is immediately apparent that $\sigma_{2} \rightarrow \pi$ in this limit, and the geodesic length inside the horizon is dominated by a single term,

$$
\begin{aligned}
L_{0} & =-\frac{L}{2} \log \left(1+\cos \sigma_{2}\right)+\cdots \\
& =-L \log \left(\cos \nu_{R}\right)+\cdots \\
& \approx 2 \pi L T t_{R}+\cdots
\end{aligned}
$$

The “..." refers to terms that remain finite as $t_{R} \rightarrow \infty$ while keeping $t_{L}$ fixed. We again find that the complexity-asvolume grows linearly with time at late times, but now at half the rate we found previously when both $t_{L}$ and $t_{R}$ progressed towards late times. We conclude that the CV conjecture makes similar predictions for $1+1$-dimensional black holes as it does in higher dimensions, provided we "by hand" multiply the geodesic length inside the horizon by a factor of the black hole entropy.

One comment on the volume calculation is that the volume of the wormhole is determined entirely by the motion of the boundary, which in turn is governed by the Schwarzian effective action. In any quantum mechanical model such as the SYK model, with a spontaneously and explicitly broken time-reparametrization symmetry, it is expected that the Schwarzian action will dominate at low energies due to the $N / \beta J$ enhancement. If the $\mathrm{CV}$ conjecture is correct, any quantum mechanical model with such a nearly conformal symmetry should exhibit a complexity that matches the above calculations in the appropriate low-energy regime. In other words, we do not expect that an improved understanding of the bulk dual of SYK will change the calculation of the volume, as long as we are not interested in exponentially late times or energies outside of the low-energy regime $\beta J \lesssim 1$. The $\mathrm{CV}$ conjecture thus suggests that the complexity in a $\mathrm{NCFT}_{1}$ is universal in the above sense. It would be interesting to understand this better from the boundary perspective.

\section{COMPLEXITY $\neq$ ACTION IN THE JT MODEL}

In this section we carry out a test of CA duality by evaluating the classical action of the JT model on a WDW patch anchored on boundary points at $\nu=\nu_{L}$ and $\nu=\nu_{R}$ as shown in Fig. 5. An immediate problem that arises is that the boundary of the WDW patch includes segments of null curves but the boundary terms in the action (3.1) involving the extrinsic curvature are only well defined if the boundary is spacelike or timelike. Another problem is that the boundary of the WDW patch fails to be everywhere smooth. The null segments that make up the boundary meet at sharp corners and the corner points will contribute to the bulk action even if they are of codimension two. Both these issues, i.e., the degeneracy of extrinsic curvature terms on null boundary segments and the corner contributions, can be addressed by applying a limiting procedure involving a family of timelike curves that approach the null boundary curves as a parameter is varied. In the following, we will adopt the prescription of Lehner et al. [16] for calculating the action on a WDW patch, which can be 


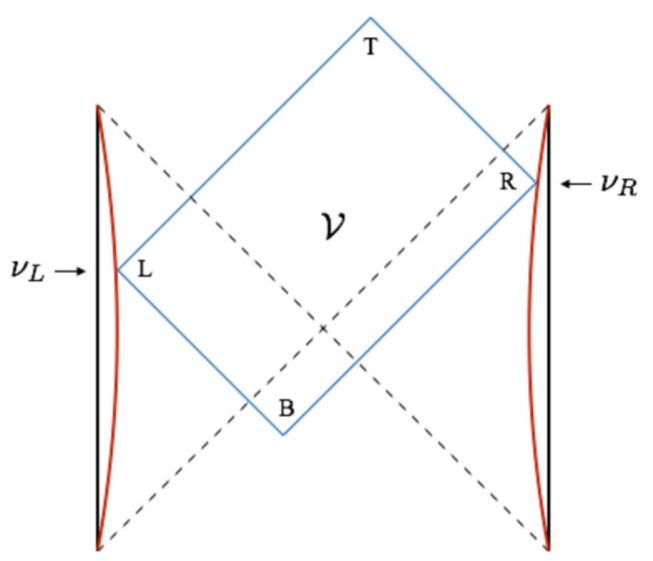

FIG. 5. Wheeler-DeWitt patch for CA calculation.

applied directly to intersecting null boundary segments and is known to be equivalent to the original results of $[7,8]$ for various black hole geometries.

The boundary of the WDW patch $\mathcal{V}$ in Fig. 5 consists of four null segments that intersect pairwise at four corner points labeled by $i \in\{L, T, R, B\}$. According to the prescription of [16], the contribution from a null boundary is given by an integral whose value depends on how the boundary curve is parametrized. The choice of boundary parametrization also affects the value of the corner contributions but when all the boundary and corner terms are added up the total is independent of parametrization. In particular, if we choose affine parametrization, so that each boundary segment is a null geodesic connecting two corner points, then the boundary integrals vanish and the action on the WDW patch consists of bulk and corner contributions only,

$$
\begin{aligned}
S_{\mathrm{WDW}}= & \frac{\varphi_{0}}{2} \int_{\mathcal{V}} d^{2} x \sqrt{-g} R+\varphi_{0} \sum_{i} a_{i} \\
& +\frac{1}{2} \int_{\mathcal{V}} d^{2} x \sqrt{-g} \varphi\left(R+\frac{2}{L^{2}}\right) \\
& +\sum_{i} \varphi_{i} a_{i},
\end{aligned}
$$

where $\varphi_{i}$ is the value of the dilaton field at the $i$ th corner point and $a_{i}= \pm \log \left|\frac{1}{2}\left(k \cdot k^{\prime}\right)\right|$, with $k$ and $k^{\prime}$ future directed tangent vectors of the null segments that intersect at the corner point in question, subject to a certain normalization condition at the JT boundary that ensures the parametrization independence of the overall result. The sign of the corner contribution $a_{i}$ associated to a given corner point is determined as follows [16]: consider either of the two null boundary segments that intersect at the corner in question. The sign is positive if the bulk region $\mathcal{V}$ lies to the future (past) of the segment and the corner point is at the past (future) end of the segment. Otherwise the sign is negative. This translates into a positive sign for the top and bottom corners in Fig. 5 and a negative sign for the left and right corners. This procedure gives an action that is invariant under the $S L(2, R)$ gauge symmetry which acts on the boundaries by an $\mathrm{AdS}_{2}$ isometry [3]. These $S L(2, R)$ transformations can be viewed as coordinate transformations, and our procedure for regulating the action (or the volume) is manifestly coordinate invariant. This is of course a desirable feature of any regulator, since complexity is a gauge-invariant quantity.

The bulk terms in the action on the WDW patch (5.1) are easily evaluated. The curvature scalar is given by $R=$ $-2 / L^{2}$ everywhere so the bulk JT term is manifestly zero. Therefore the only bulk contribution comes from the topological term and the integral over the WDW patch gives

$$
\begin{aligned}
S_{\text {bulk }}= & -\varphi_{0} \int_{\mathcal{V}} d \nu d \sigma \frac{1}{\sin ^{2} \sigma}=2 \varphi_{0}\left(\log \left(\sin \sigma_{L}\right)\right. \\
& \left.+\log \left(\sin \sigma_{R}\right)-\log \left(\sin \sigma_{T}\right)-\log \left(\sin \sigma_{B}\right)\right) .
\end{aligned}
$$

The corner terms are also easily evaluated once we settle on a choice of parametrization for each null boundary segment $(\nu(\lambda), \sigma(\lambda))$. In general the parametrization can be arbitrary but for reasons explained above we will assume $\lambda$ is an affine parameter. It is easy to check that a given boundary segment is indeed a geodesic if the components of its (future directed) tangent vector have the form

$$
\frac{d \nu}{d \lambda}= \pm \frac{d \sigma}{d \lambda}=A \sin ^{2} \sigma(\lambda)
$$

for some normalization constant $A>0$. Different values of $A$ correspond to the usual freedom to rescale the affine parameter. The prescription of [16] is to impose a normalization condition on the tangent vector involving its inner product with the timelike Killing vector that generates Schwarzschild time translations at the JT boundary,

$$
\left(\frac{d \nu}{d \lambda}, \frac{d \sigma}{d \lambda}\right) \cdot\left(\frac{d \nu}{d t}, \frac{d \sigma}{d t}\right)=-c,
$$

where $c>0$ is a constant. The inner product is to be evaluated at the corner point where the boundary segment under consideration meets the JT boundary. It is important that $c$ is time independent, i.e., that the same value of $c$ is chosen when normalizing boundary tangent vectors for WDW patches anchored at different boundary times, but $c$ is otherwise a free parameter. As it turns out, the formulas below simplify for $c=L$ and we will choose this value throughout. The normalization constant in (5.3) is then given by

$$
A=\left(L \frac{d \nu_{L}}{d t_{L}}\right)^{-1} \quad \text { or } \quad A=\left(L \frac{d \nu_{R}}{d t_{R}}\right)^{-1},
$$


depending on whether the boundary segment in question intersects the left- or right-hand boundary in Fig. 5, and the corner factors $a_{i}$ in (5.1) reduce to

$$
\begin{aligned}
& a_{L}=-2 \log \left(\sin \sigma_{L}\right)+2 \log \left(\frac{d \nu_{L}}{d t_{L}}\right), \\
& a_{R}=-2 \log \left(\sin \sigma_{R}\right)+2 \log \left(\frac{d \nu_{R}}{d t_{R}}\right), \\
& a_{T}=2 \log \left(\sin \sigma_{T}\right)-\log \left(\frac{d \nu_{L}}{d t_{L}} \frac{d \nu_{R}}{d t_{R}}\right), \\
& a_{B}=2 \log \left(\sin \sigma_{B}\right)-\log \left(\frac{d \nu_{L}}{d t_{L}} \frac{d \nu_{R}}{d t_{R}}\right) .
\end{aligned}
$$

The total contribution to the action from the corners of the WDW patch is

$$
S_{\text {corners }}=\varphi_{0} \sum_{i} a_{i}+\sum_{i} \varphi_{i} a_{i} .
$$

The first sum comes from the topological part of the action (3.1) while the second comes from the dynamical part.

The sum over the topological corner terms evaluates to

$$
\begin{aligned}
\varphi_{0} \sum_{i} a_{i}= & 2 \varphi_{0}\left(-\log \left(\sin \sigma_{L}\right)-\log \left(\sin \sigma_{R}\right)\right. \\
& \left.+\log \left(\sin \sigma_{T}\right)+\log \left(\sin \sigma_{B}\right)\right),
\end{aligned}
$$

which precisely cancels the topological bulk contribution (5.2).

This leaves us with the very simple result that the sole contribution to the WDW patch action comes from the sum over nontopological corner terms in (5.7). Let us first consider the left and right corners, which are located on the JT boundary, where the dilaton takes the value $\varphi=\varphi_{B}$ independent of the boundary time. It turns out that the corner factors $a_{L, R}$ are also time independent at leading order in $\varepsilon$ and thus the overall contribution from the leftand right-hand corners to the WDW action is constant in time. ${ }^{4}$ To see this, we first use (3.11) to evaluate the Jacobian factor between global time and Schwarzschild time,

$$
\frac{d \nu_{L, R}}{d t_{L, R}}=2 \pi T \cos \nu_{L, R}
$$

and then use that $\sin \sigma_{L, R}=\varepsilon \cos \nu_{L, R}$ on the JT boundary to find

$$
a_{L, R}=2 \log \left(\frac{4 \pi T}{\varepsilon}\right)
$$

\footnotetext{
${ }^{4}$ This is in line with the general expectation that the contribution to the WDW patch action from outside the horizon does not depend on the boundary time at which the WDW patch is anchored.
}

Finally, we consider the top and bottom corners. Working to leading order in $\varepsilon$ one finds that

$$
\sigma_{T}=\frac{\pi}{2}-\frac{\nu_{l}}{2}+\frac{\nu_{R}}{2}, \quad \sigma_{B}=\frac{\pi}{2}+\frac{\nu_{l}}{2}-\frac{\nu_{R}}{2},
$$

and then a short calculation gives

$a_{T}=a_{B}=2 \log \cos \left(\frac{\nu_{L}-\nu_{R}}{2}\right)-\log \left(\frac{d \nu_{L}}{d t_{L}} \frac{d \nu_{R}}{d t_{R}}\right)$.

It follows that both the top and bottom corner factors grow linearly with time,

$$
a_{T, B} \approx 2 \pi L T t_{L, R}+\cdots,
$$

as $t_{L, R} \rightarrow \infty$. However, their combined contribution to the WDW patch action cancels at late times due to the dilaton prefactors in (5.7). To see this, consider the WDW patch in Fig. 5 and let either $t_{L}$ or $t_{R}$ approach infinity; i.e., let $\nu_{L} \rightarrow \frac{\pi}{2}$ or $\nu_{R} \rightarrow \frac{\pi}{2}$. Either way, the top corner of the WDW patch will approach a point on the inner horizon, where $\phi=-\phi_{H}$, while the bottom corner approaches a point on the outer horizon, where $\phi=+\phi_{H}$. The leading linear growth thus cancels between the top and bottom corners.

Bringing everything together we reach the conclusion that the action on a WDW patch in Jackiw-Teitelboim gravity does not grow linearly with time at late boundary times but instead approaches a constant value. This is a surprising result in view of the conjectured duality between low-energy sectors of JT gravity and the SYK model. In the latter, quantum complexity is expected to grow linearly for a very long time. The JT model prediction for complexity is also at odds with (2.7), which gives the rate of growth of the action on a WDW patch of a Reissner-Nordström black hole in 3+1-dimensional Einstein-Maxwell theory. The discrepancy can be resolved but only after a careful reexamination of how JT gravity is obtained from the higher-dimensional theory, to which we now turn.

\section{JT GRAVITY FROM DIMENSIONAL REDUCTION}

Consider the Reissner-Nordström black holes described in Sec. II. In the near-extremal limit, the throat has approximately constant width and is very long, and supports a lowenergy sector of radial excitations that is governed by an effective two-dimensional theory. This effective theory is JT gravity $[17,18]$. To derive the action for JT gravity from the $3+1$-dimensional Einstein-Maxwell theory, Navarro-Salas and Navarro integrated out the transverse directions [17]. We review that reduction here. ${ }^{5}$

\footnotetext{
${ }^{5}$ An alternative derivation of JT gravity via dimensional reduction from a higher-dimensional Einstein-Maxwell-Dilaton theory is presented in [19].
} 
The first step is to adopt an ansatz for a spherically symmetric metric,

$$
d s^{2}=\frac{1}{\sqrt{2 \Phi}} g_{\alpha \beta} d x^{\alpha} d x^{\beta}+2 \ell^{2} \Phi d \Omega^{2},
$$

and insert it into the $3+1$-dimensional action (2.1). Here $g_{\alpha \beta}\left(x^{0}, x^{1}\right)$ is a $1+1$-dimensional metric and the dilaton $\Phi\left(x^{0}, x^{1}\right)$ is a scalar field that describes how the area of the transverse two-sphere depends on time and radial position. The resulting action is

$$
\begin{aligned}
\mathcal{S}_{2 d}= & \frac{1}{2} \int d^{2} x \sqrt{-g}\left(\Phi R+\frac{1}{\ell^{2}}(2 \Phi)^{-\frac{1}{2}}-\frac{\ell^{2}}{2}(2 \Phi)^{\frac{3}{2}} F_{\alpha \beta} F^{\alpha \beta}\right) \\
& +\int d y^{0} \sqrt{-\gamma_{00}}\left(\Phi K-\frac{1}{\ell}(2 \Phi)^{\frac{1}{4}}\right)
\end{aligned}
$$

with the boundary terms evaluated along a timelike boundary with induced metric $\gamma_{00}$. The two-dimensional field strength $F_{\alpha \beta}$ is inherited unchanged from the $3+1$ dimensional theory but the contraction in the $F^{2}$ term in the action is now with the two-dimensional metric. The $\Phi$ dependent prefactor in front of $g_{\alpha \beta}$ in (6.1) implements a Weyl transformation on the two-dimensional metric that eliminates derivative terms involving $\Phi$ from (6.2). Under spherical reduction, the extrinsic curvature term in the original $3+1$-dimensional action (2.1) gives rise to the boundary term containing the one-dimensional extrinsic curvature in (6.2) and also a term involving the normal derivative of the dilaton field on the boundary. This latter term cancels against a total derivative term involving the dilaton that comes from the $3+1$-dimensional Ricci scalar. The last term in (6.2) comes from the spherical reduction of the $K_{0}$ regulator term in the original action. Finally, if the electromagnetic boundary term (2.2) is included in the $3+1$-dimensional action, then the 1+1-dimensional action will include its spherical reduction,

$$
\mathcal{S}_{b, 2 d}^{\mathrm{em}}=\ell^{2} \int d y^{0} \sqrt{-\gamma_{00}}(2 \Phi)^{\frac{3}{2}} \hat{n}_{\alpha} F^{\alpha \beta} A_{\beta},
$$

as an additional boundary term.

The field equations of the $1+1$-dimensional theory are

$$
\begin{gathered}
0=\nabla_{\alpha}\left(\Phi^{3 / 2} F^{\alpha \beta}\right), \\
0=R-\frac{1}{\ell^{2}}(2 \Phi)^{-3 / 2}-\frac{3}{2} \ell^{2}(2 \Phi)^{1 / 2} F^{2}, \\
0=\nabla_{\alpha} \nabla_{\beta} \Phi-g_{\alpha \beta}\left(\nabla^{2} \Phi-\frac{1}{2 \ell^{2}}(2 \Phi)^{-1 / 2}\right) \\
+\ell^{2}(2 \Phi)^{3 / 2}\left(F_{\alpha \gamma} F_{\beta}^{\gamma}-\frac{1}{4} g_{\alpha \beta} F^{2}\right) .
\end{gathered}
$$

The Maxwell equation determines the electromagnetic field strength in terms of the dilaton,

$$
F_{\alpha \beta}=\frac{Q}{\ell^{2}}(2 \Phi)^{-3 / 2} \varepsilon_{\alpha \beta},
$$

where $\varepsilon_{\alpha \beta}$ is the two-dimensional Levi-Cività tensor, ${ }^{6}$ and this can be used to eliminate $F_{\alpha \beta}$ from the remaining field equations,

$$
\begin{gathered}
0=R-\frac{1}{\ell^{2}}(2 \Phi)^{-3 / 2}+\frac{3 Q^{2}}{\ell^{2}}(2 \Phi)^{-5 / 2}, \\
0=\nabla_{\alpha} \nabla_{\beta} \Phi-g_{\alpha \beta}\left(\nabla^{2} \Phi-\frac{1}{2 \ell^{2}}(2 \Phi)^{-1 / 2}+\frac{Q^{2}}{2 \ell^{2}}(2 \Phi)^{-3 / 2}\right) .
\end{gathered}
$$

We note that these equations are satisfied by the dimensional reduction of the Reissner-Nordström solution, (2.3),

$$
\begin{aligned}
d s^{2}= & -\left(\sqrt{\frac{2 x}{\ell}}-2 \ell M+Q^{2} \sqrt{\frac{\ell}{2 x}}\right) d t^{2} \\
& +\frac{d x^{2}}{\left(\sqrt{\frac{2 x}{\ell}}-2 \ell M+Q^{2} \sqrt{\frac{\ell}{2 x}}\right)},
\end{aligned}
$$

and a linear dilaton field $\Phi(x)=x / \ell$. The results of Appendix B on charged black hole thermodynamics can be reproduced from the 1+1-dimensional Euclidean onshell action, evaluated on this solution. In particular, the presence or absence of the Euclidean counterpart to the spherically reduced electromagnetic boundary term (6.3) determines whether the ensemble is at a fixed chemical potential or fixed charge.

In the following, we will mainly be interested in nearextremal black holes. More specifically, we want to study the near horizon physics of a near-extremal black hole. For this purpose, we expand the dilaton field around its value at the horizon of an extremal black hole,

$$
\Phi=\frac{Q^{2}}{2}+\varphi,
$$

and work order by order in $\varphi / Q^{2}$. At leading order, the field equations (6.8) and (6.9) reduce to

$$
\begin{gathered}
0=R+\frac{2}{Q^{3}} \\
0=\nabla_{\alpha} \nabla_{\beta} \varphi-g_{\alpha \beta}\left(\nabla^{2} \varphi-\frac{1}{Q^{3}} \varphi\right),
\end{gathered}
$$

\footnotetext{
${ }^{6}$ With the convention $\varepsilon_{01}=+\sqrt{-g}$.
} 
which are precisely the field equations (3.2) and (3.3) of the JT model with $L \equiv Q^{3 / 2} \ell$. It immediately follows that in the near-horizon region the $1+1$-dimensional radial geometry is that of $\mathrm{AdS}_{2}$, with a characteristic length scale $L$ that is parametrically large compared to the $3+1$-dimensional Planck length when $Q \gg 1$. This is the long throat of the near-extremal Reissner-Nordström black hole referred to in Sec. II.

We arrived at the reduced set of field equations by using the $1+1$-dimensional Maxwell equations to eliminate $F_{\alpha \beta}$ and it is natural to ask if the Jackiw-Teitelboim action (3.1) can similarly be obtained by integrating out the gauge field from the spherically reduced action and considering the nearhorizon limit. The answer is yes but with a somewhat subtle twist. The most naive approach, where one simply inserts the solution (6.7) for $F_{\alpha \beta}$ into the full $1+1$-dimensional action (6.2), does not work. This naive procedure does lead to a dilaton gravity theory but one where the term in the effective potential for the dilaton that comes from the gauge field has the wrong sign to reproduce the Jackiw-Teitelboim theory in the near-horizon limit. The problem can be traced to the fact that the gauge field we are integrating out is an electric field and we are replacing its kinetic energy by an effective potential for the dilaton. In fact, this kind of sign flip occurs any time a dynamical variable carrying kinetic energy is integrated out in favor of a potential energy term.

We illustrate this effect in Appendix A using the familiar example of a nonrelativistic particle moving in a central potential. The analysis of particle orbits is facilitated by introducing an effective potential for radial motion with a centrifugal term involving the conserved angular momentum. This is usually done at the level of the equations of motion but if one instead attempts to integrate out the angular variable at the level of the Lagrangian before deriving the radial equation then an analogous sign issue arises. The remedy, both for motion in a central potential and in the case at hand, is to include appropriate boundary terms for the kinetic variable in the original action. Adding a boundary term involving the gauge field does not change its dynamical equations, i.e., the Maxwell equations are not affected, but a boundary term will in general contribute to the effective dilaton potential that results from integrating out the gauge field.

As it turns out, we have already introduced a boundary term (6.3) that has the desired effect. To see this, we can use the divergence theorem to rewrite the boundary term as a $1+1$-dimensional bulk term involving a total derivative, apply the chain rule, and then use the Maxwell equation (6.4) to simplify the result:

$$
\begin{aligned}
\mathcal{S}_{b, 2 d}^{\mathrm{em}} & =\ell^{2} \int d^{2} x \sqrt{-g} \nabla_{\alpha}\left((2 \Phi)^{\frac{3}{2}} F^{\alpha \beta} A_{\beta}\right) \\
& =\frac{\ell^{2}}{2} \int d^{2} x \sqrt{-g}(2 \Phi)^{\frac{3}{2}} F^{\alpha \beta} F_{\alpha \beta} .
\end{aligned}
$$

This has the same form as the electromagnetic bulk term in the $1+1$-dimensional action that we obtained from spherical reduction but has a coefficient in front that is twice as large and of opposite sign. This is precisely what is needed to reverse the sign of the electromagnetic contribution to the dilaton effective potential when we insert the solution (6.7) for the Maxwell field into the action. The resulting bulk effective action is

$\mathcal{S}_{\text {bulk }}=\frac{1}{2} \int d^{2} x \sqrt{-g}\left(\Phi R+\frac{1}{\ell^{2}}(2 \Phi)^{-\frac{1}{2}}-\frac{Q^{2}}{\ell^{2}}(2 \Phi)^{-\frac{3}{2}}\right)$.

To capture the near-horizon physics of a near-extremal black hole we write the dilaton as in (6.11) and work order by order in $\varphi$,

$$
\mathcal{S}_{\text {bulk }}=\frac{Q^{2}}{4} \int d^{2} x \sqrt{-g} R+\frac{1}{2} \int d^{2} x \sqrt{-g} \varphi\left(R+\frac{2}{L^{2}}\right)+\cdots,
$$

This agrees precisely with the bulk terms in the JackiwTeitelboim action (3.1) if we make the identification $\varphi_{0}=\frac{Q^{2}}{2}$. The $\ldots$ denotes terms that are suppressed in the near-horizon region where $\varphi \ll Q^{2}$. Further away from the horizon the additional terms are no longer small and this simple truncation does not apply. We can ensure that we stay inside the region of interest by introducing a boundary inside the $\mathrm{AdS}_{2}$ throat region, where (6.16) remains valid, and supplementing the bulk terms by appropriate boundary terms. The boundary terms in (6.2) are evaluated in the asymptotic region far outside the $\mathrm{AdS}_{2}$ throat so we have to look elsewhere for a good definition of the boundary terms inside the throat. A Gibbons-Hawking boundary term involving the extrinsic curvature of the boundary curve is needed in order to have a well-posed variational problem for the $1+1$-dimensional metric. The further requirement that the Euclidean on-shell action give a finite free energy is satisfied by including an additional boundary term. The final form of the $1+1$-dimensional action of the nearhorizon dilaton gravity theory, including the boundary terms, is then precisely the JT action (3.1). The boundary is placed along a curve of constant dilaton, $\left.\varphi\right|_{\partial M}=\varphi_{B}$. The requirement that the boundary be inside the near-extremal $\mathrm{AdS}_{2} \times S^{2}$ region translates into $\varphi_{B} \ll Q^{2}$.

\section{COMPLEXITY = ACTION RESTORED IN THE JT MODEL}

We now return to our test of CA duality. In Sec. V we evaluated the JT action on a 1+1-dimensional WDW patch and found that it does not exhibit the expected linear growth at late times but instead approaches a constant value. This discrepancy can be traced to the procedure by 
which the JT model is obtained from higher-dimensional Einstein-Maxwell theory via dimensional reduction. The particular step we have in mind is where an electromagnetic boundary term had to be introduced in order to get the correct sign for the dilaton potential when integrating out the 1+1-dimensional Maxwell field. The boundary term does not affect the field equations of the theory but it does change the value of the action itself and a choice has to be made whether to include it when calculating the action of a WDW patch. Our calculation in Sec. V is based on the JT action (3.1) and thus includes the boundary term in question. The calculation can easily be repeated without the boundary term included. Rather than starting from scratch, we can simply evaluate the contribution from the electromagnetic boundary term on the same WDW patch and subtract it from our previous answer for the JT model. The most convenient way to proceed is to work with the equivalent bulk form (6.14) and evaluate it on shell using the $\mathrm{AdS}_{2}$ metric (3.4) with $L^{2}=Q^{3} \ell^{2}$ and the identification $\varphi_{0}=Q^{2} / 2$,

$$
\begin{aligned}
\mathcal{S}_{\mathrm{WDW}} & =\mathcal{S}_{\mathrm{WDW}}^{\mathrm{JT}}+\frac{Q^{2}}{\ell^{2}} \int_{\mathcal{V}} d^{2} x \sqrt{-g}(2 \Phi)^{-3 / 2} \\
& =-4 \varphi_{0} \log \left(\cos \nu_{L}\right)-4 \varphi_{0} \log \left(\cos \nu_{R}\right)+\cdots .
\end{aligned}
$$

The "..." denotes subleading terms that do not grow in the late-time limit. The holographic complexity thus grows linearly with time at late times when the electromagnetic boundary term is omitted,

$$
\begin{aligned}
\left.\frac{d \mathcal{S}_{\mathrm{WDW}}}{d t_{L, R}}\right|_{t_{L, R} \rightarrow \infty} & =8 \pi \varphi_{0} T \\
& =4 S T+O\left(T^{2}\right) .
\end{aligned}
$$

Furthermore, the late-time growth rate matches the known result (2.7) for a near-extremal Reissner-Nordström black hole in $3+1$ dimensions, to leading order at low temperature.

Adding a total derivative to an action does not change the equations of motion but can still affect the physics. Boundary terms can, for instance, implement a change of thermodynamic ensemble when evaluating the free energy via the Euclidean on-shell action. We have demonstrated in this paper that the complexity is another physical quantity affected by boundary terms. The action of the JT theory, as it is usually written, descends from a higherdimensional Einstein-Maxwell theory that inadvertently includes a boundary term that implies impermeable rather than permeable boundary conditions. For many purposes this is not a problem, but for calculating holographic complexity it is: we find that the CA conjecture is not consistent with the standard JT action. However, when we restore the two-dimensional Maxwell field and remove the spurious boundary term then we get the correct answer for charged black holes and, it seems reasonable to believe, SYK.

Fixing the electric field is tantamount to introducing an obstruction to the flow of charge, whereas fixing the vector potential (equivalent to fixing the chemical potential) makes the boundary permeable. It is unphysical to impose boundary conditions on a Wheeler-DeWitt patch that inhibit the flow of charge across what is essentially an arbitrary internal boundary. As we have seen with the JT model, imposing an unphysical internal boundary condition gives an unphysical result for the complexity growth.

\section{CONCLUSION}

New physics often fixes ambiguities in the action. What was once a redundancy of the description becomes physically meaningful. For example, in nongravitational physics adding a constant to the Lagrangian density makes no difference as the absolute zero of energy is unobservable. But all energy gravitates, and so in gravitational physics additive constants matter. Similarly, in classical physics the action is ambiguous with respect to multiplication by a constant. In quantum mechanics, on the other hand, multiplying the action by a constant changes $\hbar$ and so changes the importance of quantum effects-multiplicative constants matter. As a third example, quantum gravity fixes topological terms in the gravitational action, because the path integral includes integrals over different topologies.

In this paper we have seen that holographic complexity fixes yet another ambiguity in the action-in holographic complexity even boundary terms that lie at unobserved internal boundaries become physically meaningful. Indeed, we saw that it was just such an internal boundary term that explained the discrepancy between the WDW action of JT gravity and the corresponding predictions of highly charged RN black holes. In integrating out the gauge field from RN black holes to arrive at the JT theory, an internal boundary term was inadvertently introduced, which is why JT gave the wrong answer for holographic complexity growth. For most purposes (e.g., calculating boundary correlation functions), this extra term in the action does not matter-for holographic complexity, it does.

This does raise the problem of how, given an action, we are to know whether it contains all the d.o.f. necessary to describe holographic complexity. At this stage, we do not really know except to say that the Einstein-Maxwell Lagrangian produces plausible answers. Since these are the only massless d.o.f. in the theory there does not seem to be much room to add anything else.

A related open question is what principles determine the calculation of the WDW action. Does the ambiguity in the choice of boundary terms have to be fixed, or is it related to some ambiguity in the complexity? Said differently, does the WDW action with the "wrong" choice of boundary terms have any complexity interpretation? 
In addition to the SYK theory, there are other known holographic descriptions of charged RN black holes. A particularly well-known example is the D1-D5 system, and its description in terms of long multiply wound strings. It would be interesting to investigate the connection between such string theoretic constructions and the SYK model. In particular, are there approximations to the D1-D5 system whose holographic dual could be described by SYK? The multiply-wound nature of the D1-D5 long string allows the string to self-intersect and leads to an effective all-to-all coupling amongst the different windings of the string. This is known to be the origin of the fast scrambling nature of such systems and is reminiscent of the SYK model.

To summarize, we calculated the late-time rate of change of action of the WDW patch of JT gravity. We found it to be zero. The CA conjecture would then predict that the rate of complexification of the holographic dual should be zero, but this makes no sense. Instead, we traced the discrepancy to the boundaries of the WDW patch, and it was there that we found the missing gates.

\section{ACKNOWLEDGMENTS}

We are grateful to Kurt Hinterbichler, Fedor Popov, Douglas Stanford, and Alexandre Streicher. We would especially like to thank Hugo Marrochio for communicating to us the results of [20]. This research was supported by the John Templeton Foundation (A. B.), by the NDSEG program (H. L.), by NSF Grant No. PHY-1720397 (H. G.), by NSF Grant No. 1316699 (L. S.), by the Icelandic Research Fund under Grant No. 163422-053 and the University of Iceland Research Fund (L. T.), and by the Simons Foundation (Y.Z.).

Note added.-The conclusions reached in this paper have also been independently reached by Goto et al. in a paper that will appear soon [20].

\section{APPENDIX A: NOT ALL ACTIONS ARE EQUAL: THE $r-\theta$ MODEL}

The sign change that we saw in the effective action for the dilaton in Sec. VI is familiar. As a particularly simple example, consider a particle in a central potential,

$$
L=\frac{\dot{r}^{2}}{2}+r^{2} \frac{\dot{\theta}^{2}}{2}-V(r) .
$$

The angular momentum $l=r^{2} \dot{\theta}$ is conserved and the Lagrangian $L$ can be expressed as

$$
L=\frac{\dot{r}^{2}}{2}+\frac{l^{2}}{2 r^{2}}-V(r) .
$$

This equation is a numerical equality but it does not mean that we can use (A2) to get the equation of motion by thinking of $\frac{l^{2}}{2 r^{2}}$ as a potential energy. It would incorrectly give the centrifugal force as attractive. To get the right equation you need to flip the sign of the $\frac{l^{2}}{2 r^{2}}$ term,

$$
L^{\prime}=\frac{\dot{r}^{2}}{2}-\frac{l^{2}}{2 r^{2}}-V(r)
$$

With the flipped sign, the Euler-Lagrange equation for $r$ yields the correct orbital motion but the actual numerical value of the action from the original Lagrangian is correctly given by the "wrong-sign" Lagrangian in (A2) and not by (A3).

This sort of thing always happens when the kinetic energy of a d.o.f. that you integrate out becomes an effective potential energy [21]. When deriving the JT model it happens because electric field energy is kinetic energy, $\vec{E}^{2}=\left(\partial_{t} \vec{A}\right)^{2}$, which becomes an effective dilaton potential when the electric field is integrated out.

In Sec. VI we saw that the required sign-flip in the dilaton potential can be achieved by adding a suitable boundary term to the action. The same is true in the $r-\theta$ model. The difference between the actions coming from (A2) and (A3) is

$$
I-I^{\prime}=\int d t \frac{l^{2}}{r^{2}}
$$

which can be written

$$
I-I^{\prime}=\int d t i \dot{\theta}
$$

Using the conservation of $l$ this gives

$$
I-I^{\prime}=l \int d \dot{\theta}=\left.l \theta\right|_{i} ^{f},
$$

which can be expressed as a boundary term,

$$
I_{B}=\left.r^{2} \theta \dot{\theta}\right|_{i} ^{f}
$$

Our calculations involving the JT model show that the complexity-as-action is sensitive to whether the action is expressed in terms of kinetic energy or potential energy. In some sense, kinetic energy computes whereas potential energy does not. When you integrate out d.o.f. you may lose track of potential vs kinetic energy, and so lose track of the true rate of computation in the system.

\section{APPENDIX B: CHARGED BLACK HOLE THERMODYNAMICS}

The free energy of a static black hole may be obtained by continuing to Euclidean signature and evaluating the Euclidean on-shell action [13]. Which free energy this gives is determined by the boundary terms in the action. 
Let us apply this method to the Reissner-Nordström solution (2.3).

In the absence of the electromagnetic boundary term (2.2) one finds

$$
\mathcal{S}_{E}=\left.\beta F\right|_{\mu}=-S+\beta M-\beta \mu Q,
$$

where $S=\pi r_{+}^{2} / \ell^{2}$ is the Bekenstein-Hawking entropy and $\mu=Q / r_{+}$is the black hole chemical potential. This is the free energy for an ensemble where the chemical potential $\mu$ is kept fixed. On the other hand, when the electromagnetic boundary term is included, a cancellation occurs and the free energy reduces to that of a fixed charge ensemble,

$$
\left.\beta F\right|_{Q}=-S+\beta M
$$

The free energy of a two-dimensional JT black hole (3.12) is related to that of a near-extremal Reissner-Nordström black hole at fixed charge rather than at fixed chemical potential. To facilitate the comparison it is useful to express the Reissner-Nordström black hole mass $M$ and entropy $S$ in terms of temperature $T$ and (fixed) charge $Q$,

$$
\begin{aligned}
M & =M_{0}+2 \pi^{2} Q^{3} \ell T^{2}+\cdots, \\
S & =S_{0}+4 \pi^{2} Q^{3} \ell T+\cdots,
\end{aligned}
$$

where $M_{0}=Q / \ell$ and $S_{0}=\pi Q^{2}$ are the mass and entropy of an extremal black hole of charge $Q$ and the "..." in each equation refers to higher order terms in $T$.

\section{APPENDIX C: MAXWELL BOUNDARY TERMS IN $3+1$ DIMENSIONS}

In this paper, we have seen the need to be careful about creating 1 + 1-dimensional Maxwell boundary terms when integrating down from $3+1$ dimensions. However, we were able to get away without considering Maxwell boundary terms in the original 3+1-dimensional action, Eq. (2.1). This is because for electric RN solutions, the $F^{2}$ bulk action on its own requires no further boundary terms to implement our desired ensemble. However, even in $3+1$ dimensions, there is a time when we need to carefully consider Maxwell boundary terms, and that is when the black holes are not electric but magnetic.

There is, on the face of it, a bit of a puzzle about how magnetic black holes fit into the complexity = action conjecture. On the one hand, one might have thought that because of electric-magnetic duality, magnetically charged black holes should complexify at the same rate as their electrically charged duals. On the other hand, since the bulk Maxwell Lagrangian changes sign under $\vec{E} \leftrightarrow \vec{B}$,

$$
F_{\mu \nu} F^{\mu \nu} \sim \vec{B}^{2}-\vec{E}^{2},
$$

it looks like electric and magnetic black holes cannot have the same rate of action growth.

The resolution is in the boundary condition for electromagnetism [8]. The form of the Einstein-Maxwell action given in Eq. (2.1), with an $F^{2}$ bulk term and no further boundary term, is appropriate to an ensemble that keeps $A_{\mu}$ fixed on the boundary. To keep the dual vector potential $\tilde{A}_{\mu}$ fixed at the boundary, as would be the ensemble appropriate for a magnetically charged black hole, we should include a boundary term. Adding the appropriate boundary term from Braden et al. [22] gives the rate of action growth for a RN black hole of magnetic charge $P$ as

$$
\frac{d S}{d\left(t_{L}+r_{R}\right)}=\frac{P^{2}}{r_{-}}-\frac{P^{2}}{r_{+}} .
$$

Comparing to Eq. (2.7), we see that the action growth of magnetic and electric black holes are related by a $Q \leftrightarrow P$ symmetry. Even though the value of the bulk action apparently breaks electric-magnetic duality, the duality is restored by boundary terms. This is analogous to the situation encountered in [23,24].
[1] R. Jackiw, Lower dimensional gravity, Nucl. Phys. B252, 343 (1985).

[2] C. Teitelboim, Gravitation and Hamiltonian structure in two space-time dimensions, Phys. Lett. 126B, 41 (1983).

[3] J. Maldacena, D. Stanford, and Z. Yang, Conformal symmetry and its breaking in two dimensional nearly anti-de Sitter space, Prog. Theor. Exp. Phys. (2016) $12 \mathrm{C} 104$.

[4] S. Sachdev and J. Ye, Gapless Spin Fluid Ground State in a Random, Quantum Heisenberg Magnet, Phys. Rev. Lett. 70, 3339 (1993).
[5] A. Kitaev, A simple model of quantum holography2015, KITP strings seminar and entanglement program, 2015, http://online.kitp.ucsb.edu/online/entangled15/.

[6] L. Susskind, Computational complexity and black hole horizons, Fortschr. Phys. 64, 24 (2016); 64, 44(A) (2016).

[7] A. R. Brown, D. A. Roberts, L. Susskind, B. Swingle, and Y. Zhao, Holographic Complexity Equals Bulk Action?, Phys. Rev. Lett. 116, 191301 (2016).

[8] A. R. Brown, D. A. Roberts, L. Susskind, B. Swingle, and Y. Zhao, Complexity, action, and black holes, Phys. Rev. D 93, 086006 (2016). 
[9] A. Almheiri and J. Polchinski, Models of $\mathrm{AdS}_{2}$ backreaction and holography, J. High Energy Phys. 11 (2015) 014.

[10] J. Engelsöy, T. G. Mertens, and H. Verlinde, An investigation of $\mathrm{AdS}_{2}$ backreaction and holography, J. High Energy Phys. 07 (2016) 139.

[11] J. Maldacena and D. Stanford, Remarks on the Sachdev-YeKitaev model, Phys. Rev. D 94, 106002 (2016).

[12] J. Maldacena, S. H. Shenker, and D. Stanford, A bound on chaos, J. High Energy Phys. 08 (2016) 106.

[13] G. W. Gibbons and S. W. Hawking, Action integrals and partition functions in quantum gravity, Phys. Rev. D 15, 2752 (1977).

[14] J. W. York, Jr., Role of Conformal Three Geometry in the Dynamics of Gravitation, Phys. Rev. Lett. 28, 1082 (1972).

[15] A. R. Brown, H. Gharibyan, A. Streicher, L. Susskind, L. Thorlacius, and Y. Zhao, Falling toward charged black holes, Phys. Rev. D 98, 126016 (2018).

[16] L. Lehner, R. C. Myers, E. Poisson, and R. D. Sorkin, Gravitational action with null boundaries, Phys. Rev. D 94, 084046 (2016).
[17] J. Navarro-Salas and P. Navarro, $\operatorname{AdS}(2) / C F T(1)$ correspondence and near extremal black hole entropy, Nucl. Phys. B579, 250 (2000).

[18] P. Nayak, A. Shukla, R. M. Soni, S. P. Trivedi, and V. Vishal, On the dynamics of near-extremal black holes, J. High Energy Phys. 09 (2018) 048.

[19] Y. Z. Li, S. L. Li, and H. Lu, Exact embeddings of JT gravity in strings and M-theory, Eur. Phys. J. C 78, 791 (2018).

[20] K. Goto, H. Marrochio, R. C. Myers, L. Queimada, and B. Yoshida, Holographic complexity equals which action?, arXiv:1901.00014.

[21] E. Dyer and K. Hinterbichler, Boundary terms, variational principles and higher derivative modified gravity, Phys. Rev. D 79, 024028 (2009).

[22] H. W. Braden, J. D. Brown, B. F. Whiting, and J. W. York, Jr., Charged black hole in a grand canonical ensemble, Phys. Rev. D 42, 3376 (1990).

[23] S. W. Hawking and S. F. Ross, Duality between electric and magnetic black holes, Phys. Rev. D 52, 5865 (1995).

[24] J. D. Brown, Duality invariance of black hole creation rates, Phys. Rev. D 56, 1001 (1997). 